\title{
Un guide douleur, avec vous, partout, sur votre smartphone
}

\section{A pain guide, with you, everywhere, on your smartphone}

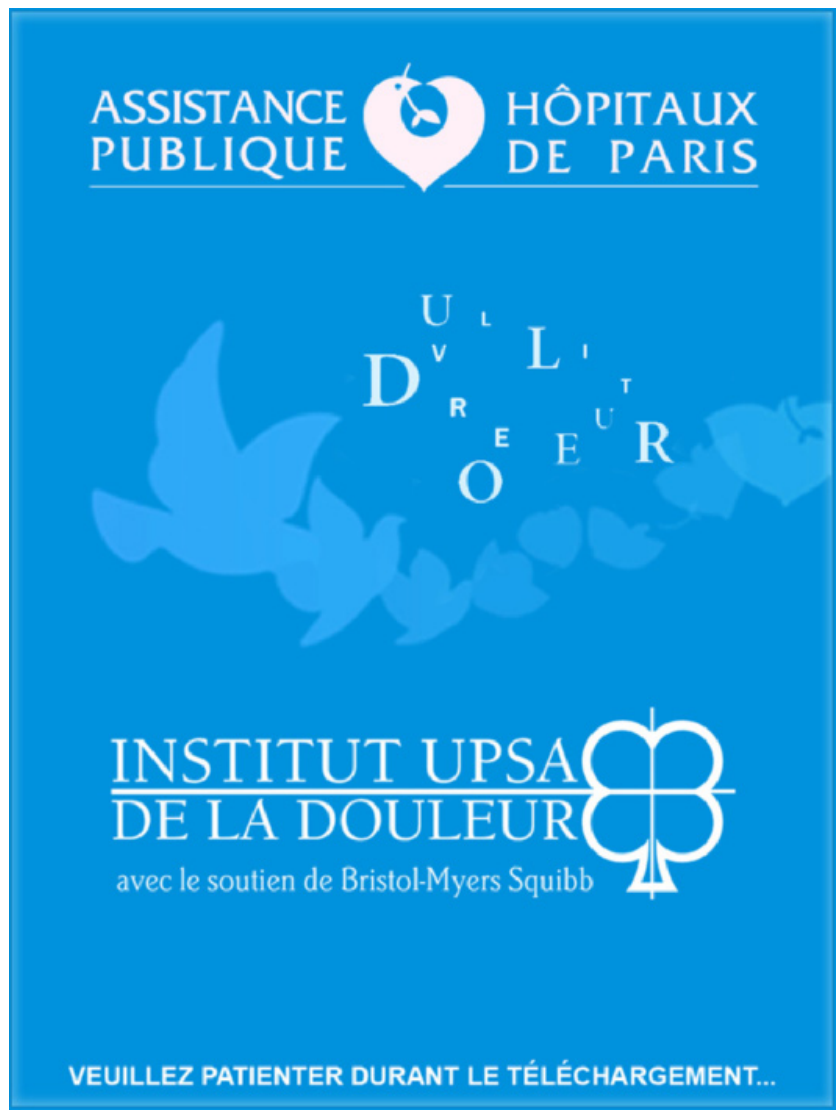

L'institut Upsa de la douleur s'est associé à l'Assistance publique-Hôpitaux de Paris pour proposer aux profession- nels de santé de télécharger gratuitement une application smartphone et tablette afin d'optimiser la prise en charge de la douleur de leurs patients.

L'application « Livret Douleur » informe sur les types de douleurs et les pathologies douloureuses comme les douleurs cancéreuses ou ostéoarticulaires et forme à la prévention des douleurs liées aux soins. Elle vise également à accompagner la gestion de la douleur en postopératoire ou aux urgences en mettant à disposition des outils pratiques tels qu'un tableau des coefficients de conversion automatique des principaux opioïdes, un panel d'échelles d'évaluation de la douleur adaptées à la typologie du patient et des échelles automatisées pour les patients âgés.

Conçue à l'initiative du comité central de lutte contre la douleur et soins palliatifs de l'Assistance publique-Hôpitaux de Paris, l'application « Livret Douleur » a été rédigée par des médecins spécialistes de différentes régions françaises ${ }^{1}$. Elle est disponible sur les plateformes de téléchargement pour iPod Touch, iPhone et iPad ainsi que sur les sites Internet de l'institut Upsa de la douleur (www.institut-upsadouleur.org) et de l'Assistance publique-Hôpitaux de Paris (http://www.aphp.fr).

Cette application a reçu le prix de la communication destinée aux spécialistes délivré par le jury du Festival de la communication santé 2011.

Pour plus d'informations sur cette application, consultez le site Internet de l'institut Upsa de la douleur : www. institut-upsa-douleur.org.

\footnotetext{
$\overline{1}$ «Livret Douleur » conçu et réalisé sous la coordination du comité central de lutte contre la douleur et soins palliatifs de l'Assistance publique-Hôpitaux de Paris — avec la participation des Drs A. Belbachir, M. Binhas, G. Abitbol, C. Bardin, E. Collin, B. George, S. Rostaing, P. Vinant, du Pr F. Bonnet, du Dr E. Guilibert, du Pr S. Perrot, des Drs F. Aubrun, H. Beloeil, du Pr H. Keita, des Drs A. Santin, C. Wood, L. Brasseur, A. Serrie, de M. G. Lebreton, de Mmes E. Malaquin-Pavant, M. Aubry, du Dr M. Galinski, avec d'autres médecins hors AP-HP : Dr G. Boccara et d'autres régions françaises : Drs R. Fuzier, E. Viel, A. Habibi.
} 